\title{
COMPARING MULTIFRACTAL FORMALISMS: THE NEIGHBORING BOXES CONDITION *
}

\author{
JULIEN BARRAL ${ }^{\dagger}$, FATHI BEN NASR ${ }^{\ddagger}$, AND JACQUES PEYRIÈRE $\$$
}

\begin{abstract}
Physicists usually compute dimensions by using boxes and they also do so when dealing with multifractals. Also in the study of some dynamical systems and multiplicative processes, boxes naturally appear. On the other hand, in geometric measure theory, it is preferred to perform computations which do not depend on a grid.

This article provides a bridge between the boxes and the grid-free approaches to the multifractal analysis of measures. Results for quasi-Bernoulli measures and statistically self-similar measures are obtained.
\end{abstract}

1. Introduction. The multifractal analysis of a measure $\mu$ aims at relating the dimension of the level sets of the pointwise Hölder exponent of $\mu$ to the Legendre transform of some kind of entropy or free energy function, a problem initially raised and studied for physical motivations $([19,17,18,25,26])$.

To define these pointwise Hölder exponents one has two alternatives: to define $\alpha(x)$ as the limit, when it exists, of either the ratio $\frac{\log \mu(B(x, r))}{\log r}$ when $r$ goes to 0 , or the ratio $\frac{\log \mu\left(Q_{n}(x)\right)}{\log \operatorname{diam}\left(Q_{n}(x)\right)}$ when $n$ goes to $+\infty$ (where $B(x, r)$ stands for the ball of radius $r$ centered at $x$ and $Q_{n}(x)$ stands for the $c$-adic box of size $c^{-n}$ which contains $x$ ). Of course the partition function is defined in terms of covers or packings by balls in the former case, by boxes in the latter case. It is usual to observe connections between these two approaches when $\mu$ possesses self-similarity properties and $\mu$ is supported by a regular enough Cantor set $([4,9,13,34,15,16,31,1])$. But there is no a priori reason why these two approaches should be connected in full generality. In this work, we give a condition ensuring that if a measure obeys the "box formalism", then it also obeys the other one. Our results apply on two families of measures supported by the full $c$-adic grid of $[0,1]$, namely the quasi-Bernoulli measures and the Mandelbrot measures.

The so called "box formalism" is better explained in the abstract setting of trees. This is the matter of the next section.

In Section 3, the Olsen multifractal formalism is recalled for the reader's convenience. The main comparison theorem is stated and proven in Section 4. This is explained in the one dimensional case, for the sake of simplicity, but at the end of this section it is said how to deal with higher dimensions. Section 5 deals with quasiBernoulli measures, and Section 6 with the Mandelbrot multiplicative measures.

*Received August 28, 2002; accepted for publication April 29, 2003.

†Projet Fractales, INRIA Rocquencourt, 78153 Le Chesnay Cedex, France

(Julien.Barral@inria.fr). This article was completed while visiting Yale University.

${ }^{\ddagger}$ Département de Mathématiques, Faculté des Sciences de Monastir, Monastir 5000, Tunisie (Fathi.BenNasr@fsm.rnu.tn).

$\S$ Yale University, Department of Mathematics, PO Box 208283, New Haven, CT 06520-8283, USA; Permanent address: Université Paris-Sud, Mathématiques bât. 425, CNRS UMR 8628, 91405 Orsay Cedex, France (Jacques.Peyriere@math.u-psud.fr). 


\section{Box analysis.}

2.1. Trees and weighted trees. Let $\mathcal{T}$ be a locally finite rooted tree. If $w$ is a node, we denote by $F(w)$ the set of nodes which immediately follow $w$. Let $\mathcal{T}_{n}$ denote the set of nodes whose geodesic distance from the root equals $n$. There is a natural topology on the set $\partial \mathcal{T}$ of geodesics stemming from the root which makes $\partial \mathcal{T}$ a metrizable totally disconnected compact space.

We identify a node of $\mathcal{T}$ with the set of geodesics going through it and the root. These sets are open and closed and they are the balls of an ultrametric distance defining the topology. A Borel measure on $\mathcal{T}$ can be identified with a function $\mu$ from the nodes of $\mathcal{T}$ to $[0,+\infty)$ subject to the condition

$$
\mu(v)=\sum_{w \in F(v)} \mu(w) \text { for all } v \in \mathcal{T} .
$$

From now on, we are given a continuous probability measure $\xi$ on $\partial \mathcal{T}$ whose support is the whole $\partial \mathcal{T}$.

If $\mu$ is a measure on $\partial \mathcal{T}$, one defines

$$
C_{n}^{\mu}(q, t)=\sum_{w \in \mathcal{T}_{n}}^{*} \mu(w)^{q} \xi(w)^{t},
$$

where the star means that the terms for which $\mu(w)=0$ are removed from the sum -a convention valid throughout this article-, and

$$
\tau_{\mu}(q)=\sup \left\{t \in \mathbb{R} \mid \limsup _{n \rightarrow \infty} C_{n}^{\mu}(q, t)=+\infty\right\} .
$$

Since $C_{n}^{\mu}$ is a log-convex function, the function $\tau_{\mu}$ is easily seen to be convex and non-increasing.

One the other hand, for any $\alpha \in \mathbb{R}$, one considers the set

$$
E_{\alpha}=\left\{\begin{array}{l|l}
x \in \partial \mathcal{T} \mid \lim _{\substack{\xi(w) \rightarrow 0 \\
x \in w}} \frac{\log \mu(w)}{\log \xi(w)}=\alpha
\end{array}\right\} .
$$

Let $\operatorname{dim}_{\xi}$ be the Hausdorff dimension defined by using $\xi(w)$ instead of the diameter of $w$ (see [8]).

Then it is known (for instance, see [11]) that, for each $q$ for which $\tau_{\mu}^{\prime}(q)$ exists, one has

$$
\operatorname{dim}_{\xi} E_{-\tau_{\mu}^{\prime}(q)} \leq \tau_{\mu}^{*}\left(-\tau_{\mu}^{\prime}(q)\right),
$$

where $\tau_{\mu}^{*}$ is the Legendre transform of $\tau_{\mu}$, i.e. $\tau_{\mu}^{*}(t)=\inf _{q \in \mathbb{R}} q t+\tau_{\mu}(q)$. Indeed, the stronger inequality [11]

$$
\operatorname{Dim}_{\xi} E_{-\tau_{\mu}^{\prime}(q)} \leq \tau_{\mu}^{*}\left(-\tau_{\mu}^{\prime}(q)\right),
$$

where $\operatorname{Dim}_{\xi}$ stands for the packing dimension (defined in [36]), is almost an instance of the Chernoff formula [12]. Of course, if these inequalities lead to negative dimensions, this means that the corresponding set is empty.

The formula

$$
\operatorname{dim}_{\xi} E_{-\tau_{\mu}^{\prime}}(q)=\tau_{\mu}^{*}\left(-\tau_{\mu}^{\prime}(q)\right)
$$


is shown [11] to hold if there exists a measure $\mu_{q}$, called a Gibbs measure, such that there exits $C>0$ such that, for all $w \in \mathcal{T}$,

$$
\frac{1}{C} \mu(w)^{q} \xi(w)^{\tau_{\mu}(q)} \leq \mu_{q}(w) \leq C \mu(w)^{q} \xi(w)^{\tau_{\mu}(q)}
$$

Indeed, the equality (2.2) appeared, in a non rigourous mathematical form, in [18] and was proven to hold for some examples [9, 13].

It was noticed in [33] that (2.1) still holds when the function $C_{n}^{\mu}$ above is replaced by the infimum of the corresponding sums on the sections of $\mathcal{T}$ which lie below level $n$ (in other terms, one considers covers of $\partial \mathcal{T}$ by ultrametric balls).

Indeed, as shown in [5], only the right hand side inequality in (2.3) is needed to prove (2.2). Since this is reminiscent of the Frostman lemma, it was natural to consider generalized Hausdorff measures: for $q$ and $t$ in $\mathbb{R}$, and $A \subset \partial \mathcal{T}$, define

$$
\mathcal{H}_{\mu}^{q, t}(A)=\lim _{\delta \searrow 0} \inf \left\{\sum \mu\left(w_{j}\right)^{q} \xi\left(w_{j}\right)^{t} \mid w_{j} \in \mathcal{T}, A \subset \bigcup w_{j}, \xi\left(w_{j}\right) \leq \delta\right\}
$$

LemMA 2.1 ([5]). If a compact set $A$ is such that $\mathcal{H}_{\mu}^{q, t}(A)>0$, then it carries a probability measure $\nu$ satisfying $\nu(w) \leq C \mu(w)^{q} \xi(w)^{t}$ for all $w$.

Then, if one defines

$$
\begin{aligned}
& \mathcal{K}_{\mu, \delta}^{q, t}=\sup \left\{\sum_{\mu} \mu\left(w_{j}\right)^{q} \xi\left(w_{j}\right)^{t} \mid w_{j} \in \mathcal{T}, \xi\left(w_{j}\right) \leq \delta, w_{j} \cap w_{k}=\emptyset \text { for } j \neq k\right\}, \\
& \mathcal{K}_{\mu}^{q, t}=\underbrace{\lim }_{\searrow 0} \mathcal{K}_{\mu, \delta}^{q, t},
\end{aligned}
$$

and

$$
\Lambda_{\mu}(q)=\sup \left\{t \in \mathbb{R} \mid \mathcal{K}_{\mu}(q, t)=+\infty\right\},
$$

this lemma implies the following theorem.

THEOREM $2.2([5])$. Suppose that $\Lambda_{\mu}^{\prime}(q)$ exists and that $\mathcal{H}_{\mu}^{q, \Lambda_{\mu}(q)}(\operatorname{supp} \mu)>0$. Then $\operatorname{dim}_{\xi} E_{-\Lambda_{\mu}^{\prime}}(q)=\Lambda_{\mu}^{*}\left(-\Lambda_{\mu}^{\prime}(q)\right)$.

2.2. Homogeneous trees. In this section, we suppose that the tree $\mathcal{T}$ is homogeneous of order $c$ and that $\xi$ is uniformly distributed. In this context, it is more convenient to see $\mathcal{T}$ as the free monoid on a $c$-letter alphabet $\mathcal{A}$.

So, let $\mathcal{A}^{*}=\bigcup_{n>0} \mathcal{A}^{n}$ be the free monoid consisting of words on $\mathcal{A}$ endowed with the concatenation. The operation of concatenation will be simply denoted by juxtaposition, and by a dot, when this is necessary for a better understanding. The empty word $\epsilon$ is the identity element. The length of a word $w$ is denoted by $|w|$. If a word $v$ is a prefix of the word $w$, we write $v \prec w$. This defines an order on $\mathcal{A}^{*}$. Endowed with this order, $\mathcal{A}$ is a tree the root of which is $\epsilon$. If $v$ and $w$ are words, $v \wedge w$ stands for their largest common prefix. The quantity $d(v, w)=c^{-|v \wedge w|}$ defines an ultrametric distance on $\mathcal{A}^{*}$.

The completion $\widehat{\mathcal{A}^{*}}$ of $\left(\mathcal{A}^{*}, d\right)$ is a compact space which is the disjoint union of $\mathcal{A}^{*}$ and $\partial \mathcal{A}^{*}$. The elements of $\partial \mathcal{A}^{*}$ can be viewed as the infinite sequences of elements of $\mathcal{A}$. The notion of prefix extends in a natural way to elements of $\partial \mathcal{A}^{*}$.

We identify $w \in \mathcal{A}^{*}$ with the cylinder $\left\{x \in \partial \mathcal{A}^{*} \mid w \prec x\right\}$, so a Borel measure $\mu$ on $\partial \mathcal{A}^{*}$ is a mapping from $\mathcal{A}^{*}$ to $\mathbb{R}^{+}$fulfilling the following compatibility condition

$$
\mu(w)=\sum_{a \in \mathcal{A}} \mu(w a) \quad \text { for all } w \in \mathcal{A}^{*} .
$$


At last, the measure $\xi$ is so defined: $\xi(w)=c^{-|w|}$.

In this setting, the $\tau_{\mu}$ function can be defined as follows.

$$
\tau_{\mu}(q)=\limsup _{n \rightarrow+\infty} \frac{1}{n} \log _{c} \sum_{w \in \mathcal{A}^{n}}^{*} \mu(w)^{q},
$$

where $q$ is a real number and the star means, as already said, that the summation runs only on the $w$ having a non-zero measure. This function $\tau_{\mu}$ is convex and nonincreasing as observed previously.

3. A centered multifractal formalism. Let $(X, d)$ be a metric space and $\mu$ a positive atomless Borel measure on $X$. The support of $\mu$ is denoted by supp $\mu$.

According to Olsen [32], we define several measures and premeasures indexed by a couple $(q, t)$ of real numbers. If $E$ is a subset of $X$ and $\delta$ is a positive real number, we set

$$
\overline{\mathcal{P}}_{\mu, \delta}^{q, t}(E)=\sup \sum^{*} \mu\left(B\left(x_{j}, r_{j}\right)\right)^{q} r_{j}^{t}
$$

this supremum being taken over the collections $\left\{B\left(x_{j}, r_{j}\right)\right\}$ of mutually disjoint balls whose centers $x_{j}$ belong to $E$ and whose radii $r_{j}$ are less than $\delta$. The star means that we omit in the summation the terms which are obviously infinite (i.e. zero raised to a negative power). As previously said, this convention holds throughout this paper. Of course, as long as the measure $\mu$ has no point masses, it does not matter that balls be open or closed. In the sequel, we deal with such measures and, it will be convenient for our reasonings to consider only closed balls.

We consider the limit

$$
\overline{\mathcal{P}}_{\mu}^{q, t}(E)=\lim _{\delta \searrow 0} \overline{\mathcal{P}}_{\mu, \delta}^{q, t}(E)
$$

The function $\overline{\mathcal{P}}_{\mu}^{q, t}$ is called packing pre-measure. It lacks $\sigma$-subadditivity to be a Caratheodory outer measure. This is why one considers the following quantity

$$
\mathcal{P}_{\mu}^{q, t}(E)=\inf _{E \subset \bigcup E_{j}} \sum \overline{\mathcal{P}}_{\mu}^{q, t}\left(E_{j}\right)
$$

which, as a function of $E$, is an outer measure. (This is the same process as for defining packing measures, which were introduced in [36].)

In a similar way, one defines Hausdorff-like measures.

$$
\overline{\mathcal{H}}_{\mu, \delta}^{q, t}(E)=\inf \sum^{*} \mu\left(B\left(x_{j}, r_{j}\right)\right)^{q} r_{j}^{t},
$$

this infimum being taken over the coverings $\left\{B\left(x_{j}, r_{j}\right)\right\}$ of $E$ by balls whose centers $x_{j}$ belong to $E$ and whose radii $r_{j}$ are less than $\delta$, and consider the limit

$$
\overline{\mathcal{H}}_{\mu}^{q, t}(E)=\lim _{\delta \searrow 0} \overline{\mathcal{H}}_{\mu, \delta}^{q, t}(E)
$$

In order to deal with an outer measure, one defines

$$
\mathcal{H}_{\mu}^{q, t}(E)=\sup _{F \subset E} \overline{\mathcal{H}}_{\mu}^{q, t}(F)
$$


These last measures are the multifractal counterparts of the centered Haudorff measures introduced in [35].

For a fixed $q$, if, for some $t$ one has $\overline{\mathcal{P}}_{\mu}^{q, t}(\operatorname{supp} \mu)<+\infty$, then, for all $t^{\prime}>t$, one has $\overline{\mathcal{P}}_{\mu}^{q, t^{\prime}}(\operatorname{supp} \mu)=0$. Therefore, there exists a unique $\Lambda_{\mu}(q) \in \overline{\mathbb{R}}$ such that $\overline{\mathcal{P}}_{\mu}^{q, t}(\operatorname{supp} \mu)$ is infinite if $t<\Lambda_{\mu}(q)$ and zero if $t>\Lambda_{\mu}(q)$. $\mathcal{H}_{\mu}^{q, t}$

In a similar way, two functions $B_{\mu}$ and $b_{\mu}$ are associated respectively to $\mathcal{P}_{\mu}^{q, t}$ and

All these three functions are non-increasing; $\Lambda_{\mu}$ and $B_{\mu}$ are convex. It is clear that $B_{\mu} \leq \Lambda_{\mu}$. If, moreover the metric space $(X, d)$ has the Besicovitch covering property defined below, one has $b_{\mu} \leq B_{\mu}$.

If $\alpha$ and $\beta$ are two real numbers such that $\alpha \leq \beta$, one considers the following sets

$$
X_{\mu}(\alpha, \beta)=\left\{x \mid \alpha \leq \liminf _{r \searrow 0} \frac{\log \mu(B(x, r))}{\log r} \leq \limsup _{r \searrow 0} \frac{\log \mu(B(x, r))}{\log r} \leq \beta\right\} .
$$

Instead of $X_{\mu}(\alpha, \alpha)$, we shall simply write $X_{\mu}(\alpha)$.

If the derivative of $B_{\mu}$ exists at point $q$, it is known [32] that the following inequalities hold, with the convention that a set of negative dimension ${ }^{1}$ is empty,

$$
\begin{aligned}
\operatorname{dim} X_{\mu}\left(-B_{\mu}^{\prime}(q)\right) & \leq b_{\mu}^{*}\left(-B_{\mu}^{\prime}(q)\right) \\
\operatorname{Dim} X_{\mu}\left(-B_{\mu}^{\prime}(q)\right) & \leq B_{\mu}^{*}\left(-B_{\mu}^{\prime}(q)\right),
\end{aligned}
$$

where, as previously, the star as an exponent denotes the Legendre transform -i.e. $f^{*}(\alpha)=\inf _{q \in \mathbb{R}} \alpha q+f(q)$ - and where dim and Dim stand for the Hausdorff and packing dimensions.

DEFINITION 3.1. If $B_{\mu}^{\prime}(q)$ exists and if all the quantities in (3.1) are equal, one says that the measure $\mu$ obeys the multifractal formalism at point $q$.

Before recalling the following theorem [6], we need another definition.

Definition 3.2. A metric space $(X, d)$ is said to have the Besicovitch covering property if there exists a positive integer $\beta$ such that, given any collection $\left\{B\left(x_{i}, r_{i}\right)\right\}_{i \in I}$ of balls, one can extract from it packings $P_{1}, P_{2}, \ldots, P_{\beta}$ which altogether form a cover of the set $\left\{x_{i}\right\}_{i \in I}$.

Any euclidean space has this property, as well as, of course, any ultrametric space.

THEOREM 3.3. If $(X, d)$ has the Besicovitch covering property, if $\alpha=-B_{\mu}^{\prime}(q)$ exists, and if $\mathcal{H}_{\mu}^{q, B_{\mu}(q)}(\operatorname{supp} \mu)>0$, then

$$
\operatorname{dim} X_{\mu}(\alpha)=\operatorname{Dim} X_{\mu}(\alpha)=B_{\mu}^{*}(\alpha) \quad \text { and } \quad b_{\mu}(q)=B_{\mu}(q) .
$$

\section{Comparing multifractal analyses.}

\subsection{Stating the problem.}

Lemma 4.1. Let $\mu$ be a non-zero positive measure on $\partial \mathcal{A}^{*}$. Then one has $\tau_{\mu}=\Lambda_{\mu}$.

Proof. We begin by noticing that, if $t>\tau_{\mu}(q)$, one has

$$
\sum_{w \in \mathcal{A}^{*}}^{*} \mu(w)^{q} c^{-t|w|}<+\infty
$$

\footnotetext{
${ }^{1}$ For a further interpretation of negative dimensions, see $[26,27]$.
} 
It results that, if $t>\tau_{\mu}(q), \overline{\mathcal{P}}_{\mu}^{q, t}(\operatorname{supp} \mu)$ is finite, and therefore $t \geq \Lambda_{\mu}(q)$. This proves $\Lambda_{\mu} \leq \tau_{\mu}$. The converse inequality is obvious.

The equality asserted in this lemma is similar to the equivalence of two different definitions of the quantity called $\Delta(E)$ in [36].

The map $\gamma$. There is a natural map from $\partial \mathcal{A}^{*}$ onto $\mathbb{R}$ : let us take $\mathcal{A}=$ $\{0,1,2, \ldots, c-1\}$ and consider the map $\gamma$ which sends the element $x=a_{1} a_{2} \cdots a_{n} \cdots$ on the number $\sum_{n \geq 1} a_{n} c^{-n}$. This map sends cylinders onto $c$-adic intervals, and when a measure $\mu$ on $\partial \mathcal{A}^{*}$ has no point masses, it is equivalent in many problems to consider it or its image $\nu=\gamma_{*}(\mu)$ under $\gamma$.

This rises the natural question of deciding when the multifractal analysis of $\nu$ (in $(\mathbb{R},||)$ ) and $\mu$ (in $\left(\partial \mathcal{A}^{*}, d\right)$ ) are linked. Indeed, there are no reasons (even when $\nu$ is doubling) why $B_{\mu}$ and $B_{\nu}$, for instance, should coincide. There are no reasons either that $X_{\nu}(\alpha, \beta)$ should be the image of $X_{\mu}(\alpha, \beta)$ under $\gamma$. Nevertheless, one has the following fact.

LeMma 4.2. One has $\Lambda_{\nu} \leq \tau_{\mu}$.

Proof. Consider first the case $q \leq 0$. Let $\left\{B\left(x_{j}, r_{j}\right)\right\}$ be a centered packing of $\operatorname{supp} \nu$, and $t>\tau_{\mu}(q)$. Each $B\left(x_{j}, r_{j}\right)$ contains a $c$-adic interval $I_{j}$ such that $x_{j} \in I_{j}$, $\left|I_{j}\right|>r_{j} / c$ (where $\left|I_{j}\right|$ stands for the length of $I_{j}$ ), and $\nu\left(I_{j}\right)>0$ (do not forget that $\left.x_{j} \in \operatorname{supp} \nu\right)$. Therefore

$$
\begin{aligned}
\sum \nu\left(B\left(x_{j}, r_{j}\right)\right)^{q} r_{j}^{t} & \leq c^{t^{+}} \sum \nu\left(I_{j}\right)^{q}\left|I_{j}\right|^{t} \\
& \leq c^{t^{+}} \sum_{w \in \mathcal{A}^{*}}^{*} \mu(w)^{q} c^{-t|w|}<+\infty
\end{aligned}
$$

where $t^{+}=\max \{t, 0\}$. This yields $\underset{\nu}{q, t}(\operatorname{supp} \nu)<\infty$. Consequently $\Lambda_{\nu}(q) \leq \tau_{\mu}(q)$.

Now, consider the case $q>0$. Let $\left\{B\left(x_{j}, r_{j}\right)\right\}$ be a centered packing of $\operatorname{supp} \nu$, and $t>\tau_{\mu}(q)$. Each $B\left(x_{j}, r_{j}\right)$ is covered by at most $c+1 c$-adic intervals $I_{j, 1}, I_{j, 2}, \ldots, I_{j, k_{j}}$ of non-zero $\nu$-measure and of common length $l$ satisfying $l \leq 2 r_{j}<c l$. One has

$$
\nu\left(B\left(x_{j}, r_{j}\right)\right)^{q} \leq k_{j}^{(q-1)^{+}} \sum_{k=1}^{k_{j}} \nu\left(I_{j, k}\right)^{q}
$$

and $r_{j}^{t} \leq 2^{-t} c^{t^{+}}|I|^{t}$. Observe also that a $c$-adic interval can appear at most twice as an $I_{j, k}$, since $\left\{B\left(x_{j}, r_{j}\right)\right\}$ is a packing. Therefore

$$
\begin{aligned}
& \sum \nu\left(B\left(x_{j}, r_{j}\right)\right)^{q} r_{j}^{t} \\
\leq & 2^{1-t}(c+1)^{(q-1)^{+}} c^{t^{+}} \sum_{w \in \mathcal{A}^{*}}^{*} \mu(w)^{q} c^{-t|w|}<+\infty .
\end{aligned}
$$

This proves again that $\Lambda_{\nu}(q) \leq \tau_{\mu}(q)$.

In the next section, we give conditions which ensure that the multifractal analysis of $\mu$ and $\nu$ are equivalent. In the subsequent sections, we analyse some examples. 
4.2. The comparison theorems. As recalled in Lemma 2.1 the inequality $\mathcal{H}_{\mu}^{q, t}(\operatorname{supp} \mu)>0$ (where $\mu$ is a measure on $\left.\partial \mathcal{A}^{*}\right)$ is equivalent to the existence of a measure $\mu_{q, t}$ with the property that, for a suitable $C>0$, one has $\mu_{q, t}(B) \leq C \mu(B)^{q}|B|^{t}$, for any ball $B$ of radius $|B|$ small enough. It is convenient to set the following definition.

DEFINITION 4.3. Let $\mu$ be a measure on a metric space $X$ and $q$ and $t$ be two real numbers. A non-zero measure $\mu^{\prime}$ having the property that there exists two positive numbers $C$ and $\eta$ such that, for all $x \in \operatorname{supp} \mu$ and $r \leq \eta$, one has $\mu^{\prime}(B(x, r)) \leq$ $C \mu(B(x, r))^{q} r^{t}$ is called a Frostman measure for $\mu$ at $(q, t)$.

From now on we stick to the convention that $\mu$ (with or without subscript) is a measure on $\partial \mathcal{A}^{*}$ and that $\nu$ (with or without subscript) stands for the corresponding image measure under $\gamma$.

As, via $\gamma$, the elements of $\mathcal{A}^{n}$ correspond to the $c$-adic intervals of length $c^{-n}$ contained in $[0,1]$, one can assign to each $w \in \mathcal{A}^{n}$ a number $\imath(w)$ so as to have $\gamma(w)=\left[\imath(w) c^{-n},(\imath(w)+1) c^{-n}\right]$. Finally, if the words $v$ and $w$ have the same length, we set $\delta(v, w)=|\imath(v)-\imath(w)|$.

If $\nu=\gamma_{*}(\mu)$ has no masses at the endpoints of $\gamma(w)$, then $\nu(\gamma(w))=\mu(w)$. But, one always has $\quad \nu(\gamma(w)) \leq \sum_{\substack{v \in \mathcal{A}^{*}(v, w) \leq 1 \\ \delta(v)}} \mu($

From now on, in this section, we deal with an atomless measure $\mu$ on $\partial \mathcal{A}^{*}$ and its image $\nu$ under $\gamma$.

Lemma 4.4. Let $q \in \mathbb{R}$ and $\eta>0$ be two numbers. Assume there exists a Frostman measure $\mu_{q}$ for $\mu$ at $\left(q, \tau_{\mu}(q)\right)$. Let $\nu_{q}=\gamma_{*}\left(\mu_{q}\right)$ stand for the image of $\mu_{q}$ under $\gamma$. Then we have the following facts.

1. When $q<0$, if $c^{-(n+1)}<2 r \leq c^{-n}$, one has

$$
\begin{gathered}
\int_{\text {supp } \nu}\left[\nu(B(x, r))^{-q} \nu_{q}(B(x, r))\right]^{\eta} d \nu_{q}(x) \leq \\
C c^{-n(1+\eta) \tau_{\mu}(q)} \sum_{\substack{v, w \in \mathcal{A}^{n} \\
\delta(v, w) \leq 3}}^{*} \mu(v)^{(1+\eta) q} \mu(w)^{-\eta q} .
\end{gathered}
$$

2. When $q \geq 0$, if $c^{-n} \leq r<c^{1-n}$, one has

$$
\begin{gathered}
\int_{\operatorname{supp} \nu}\left[\nu(B(x, r))^{-q} \nu_{q}\left(B\left(x,\left(1+\frac{1}{c}\right) r\right)\right)\right]^{\eta} d \nu_{q}(x) \leq \\
C c^{-n(1+\eta) \tau_{\mu}(q)} \sum_{\substack{v, w \in \mathcal{A}^{n} \\
\delta(v, w) \leq 4 c+2}}^{*} \mu(v)^{(1+\eta) q} \mu(w)^{-\eta q} .
\end{gathered}
$$

In both cases the constant $C$ depends only on $q, \eta$, and $c$.

Proof. We begin by assuming $q<0$. By the Besicovitch covering lemma, we extract from the collection $\{B(x, r)\}_{x \in \operatorname{supp} \nu}$ of balls $\beta$ (a universal constant) packings which altogether cover $\operatorname{supp} \nu$.

One has

$$
\begin{aligned}
\int_{B(a, r) \cap \operatorname{supp} \nu}\left[\nu(B(x, r))^{-q} \nu_{q}(B(x, r))\right]^{\eta} d \nu_{q}(x) \leq \\
\\
\nu(B(a, 2 r))^{-\eta q} \nu_{q}(B(a, 2 r))^{1+\eta}
\end{aligned}
$$


The interval $B(a, 2 r)$ is contained in at most two $c$-adic consecutive intervals $I_{1}$ and maybe $I_{2}$ of common length $l$ such that $l / c<2 r \leq l$. We add to this collection of one or two intervals the two $c$-adic intervals of the same length which are contiguous. We keep only the ones having a non-zero $\nu$-measure. Then we have intervals $J_{1}, J_{2}$, $J_{3}$, and maybe $J_{4}$ such that, if $I$ is any of the intervals $I_{1}$ or $I_{2}, \nu_{q}(I)$ is majorized by the sum $\sum \mu_{q}\left(J_{i}\right)$. We had to adjunct the right and left intervals to cope with the case where $\nu_{q}$ has masses at some $c$-adic points. In these conditions, we have

$$
\begin{aligned}
& \nu(B(a, 2 r))^{-\eta q} \nu_{q}(B(a, 2 r))^{1+\eta} \leq C_{1}\left(\sum \nu\left(J_{j}\right)^{-q \eta}\right)\left(\sum \nu_{q}\left(J_{j}\right)^{1+\eta}\right) \\
& \leq C_{1}\left(\sum \nu\left(J_{j}\right)^{-q \eta}\right)(\left.\sum \nu\left(J_{j}\right)^{(1+\eta) q}\left|J_{j}\right|^{(1+\eta) \tau_{\mu}(q)}\right) \\
& \leq C_{2} c^{-n(1+\eta) \tau_{\mu}(q)} \sum \nu\left(J_{i}\right)^{-q \eta} \nu\left(J_{j}\right)^{(1+\eta) q}
\end{aligned}
$$

where the constants $C_{1}$ and $C_{2}$ depend only on $c, q$, and $\eta$.

Now, consider the case $q \geq 0$. For every $x \in \operatorname{supp} \nu, B(x, r)$ contains at least one $c$-adic interval $I_{x}$ of length $c^{-n}$ such that $\mu\left(I_{x}\right)>0$ and $c^{-n} \leq r<c^{1-n}$.

Fix $a \in \operatorname{supp}(\mu)$ and consider the set of closed $c$-adic intervals of length $c^{-n}$ the interior of which intersect $B\left(a,\left(2+\frac{1}{c}\right) r\right)$, and, as previously, add to them two intervals of the same generation, one on the right, the other on the left. 'The number of elements of the set $S$ of these intervals is bounded by $4 c+3$. Therefore

$$
\begin{gathered}
\int_{B(a, r) \cap \operatorname{supp} \nu}\left[\nu(B(x, r))^{-q} \nu_{q}\left(B\left(x, \frac{(c+1) r}{c}\right)\right)\right]^{\eta} d \nu_{q}(x) \\
\leq \int_{B(a, r) \cap \operatorname{supp} \nu}\left[\nu^{-q}\left(I_{x}\right) \nu_{q}\left(B\left(x, \frac{(c+1) r}{c}\right)\right)\right]^{\eta} d \nu_{q}(x) \\
\leq\left[\sum_{I \in S} \nu^{-q}(I)\right]^{\eta} \int_{B(a, r) \cap \operatorname{supp} \nu} \nu_{q}\left(B\left(x, \frac{(c+1) r}{c}\right)\right)^{\eta} d \nu_{q} \\
\leq\left[\sum_{I \in S} \nu^{-q}(I)\right]^{\eta} \nu_{q}\left(B\left(a,\left(2+\frac{1}{c}\right) r\right)\right)^{1+\eta} \\
\leq C\left[\sum_{I \in S} \nu^{-q \eta}(I)\right]\left[\sum_{I \in S} \nu_{q}(I)^{1+\eta}\right] .
\end{gathered}
$$

The proof ends as previously.

Definition 4.5 (NBC). A measure $\mu$ on $\partial \mathcal{A}^{*}$ is said to satisfy the Neighboring Boxes Condition (NBC) for $q \in \mathbb{R}$ if, for all $\varepsilon>0$, there exists $\eta>0$ such that

$$
\sum_{n>0} c^{-n\left(\tau_{\mu}(q)+\varepsilon \eta\right)} \sum_{\substack{v, w \in \mathcal{A}^{n} \\ \delta(v, w) \leq c^{\prime}}} \mu(v)^{-\eta q} \mu(w)^{(1+\eta) q}<+\infty
$$

where $c^{\prime}=3$ if $q<0, c^{\prime}=4 c+2$ otherwise. 
Lemma 4.6. Let $q \in \mathbb{R}$. Assume there exists a Frostman measure $\mu_{q}$ for $\mu$ at $\left(q, \tau_{\mu}(q)\right)$ and that $\mu$ fulfills the $N B C$ for $q$. Then, we have $b_{\nu}(q)=\tau_{\mu}(q)$.

Also, there exists a constant $C>0$ such that, for any $\varepsilon>0$, for $\nu_{q}$-almost every $x$, and for $r$ small enough, $\nu_{q}(B(x, r)) \leq C \nu(B(x, r))^{q} r^{\tau_{\mu}(q)-\varepsilon}$.

Proof. First consider the case $q<0$. Set

$$
F(r)=\int_{\operatorname{supp} \nu}\left[\nu(B(x, r))^{-q} \nu_{q}(B(x, r))\right]^{\eta} d \nu_{q}(x) .
$$

For any $\varepsilon>0$, we have

$$
\sum_{n \geq 0} F\left(c^{-n}\right) c^{n \eta\left(\tau_{\mu}(q)-\varepsilon\right)} \leq C \sum_{n \geq 0} c^{-n\left(\tau_{\mu}(q)+\eta \varepsilon\right)} \sum_{\substack{v, w \in \mathcal{A}^{n} \\ \delta(v, w) \leq c^{\prime}}} \mu(v)^{-\eta q} \mu(w)^{(1+\eta) q},
$$

due to Lemma 4.4. The right hand side quantity is finite for a suitable choice of $\eta$, because of the NBC.

As a consequence of the Borel-Cantelli lemma, for $\nu_{q}$-almost every $x$, one has

$$
\nu_{q}\left(B\left(x, c^{-n}\right)\right) \leq \nu\left(B\left(x, c^{-n}\right)\right)^{q} c^{-n\left(\tau_{\mu}(q)-\varepsilon\right)}
$$

for $n$ large enough. Consequently for $\nu_{q}$-almost every $x$, one has $\nu_{q}(B(x, r)) \leq$ $C \nu(B(x, r))^{q} r^{\tau_{\mu}(q)-\varepsilon}$ for $r$ small enough.

Similarly, when $q \geq 0$, for $\nu_{q}$-almost every $x$, one has

$$
\nu_{q}\left(B\left(x, \kappa^{1-n}\right)\right) \leq C \nu\left(B\left(x, \kappa^{-n}\right)\right)^{q} \kappa^{-n\left(\tau_{\mu}(q)-\varepsilon\right)}
$$

for $n$ large enough, where $\kappa=1+1 / c$. This implies that, for $\nu_{q}$-almost every $x$, one has $\nu_{q}(B(x, r)) \leq C \nu(B(x, r))^{q} r^{\tau_{\mu}(q)-\varepsilon}$ for $r$ small enough. $\tau_{\mu}(q)$.

It results from these estimates that $\mathcal{H}_{\nu}^{q, \tau_{\mu}(q)-\varepsilon}(\operatorname{supp} \nu)>0$, and therefore $b_{\nu}(q) \geq$

LEMMA 4.7. Under the same assumptions as in Lemma 4.6, one has

$$
\nu_{q}\left(\mathbb{R} \backslash X_{\nu}\left(-B_{\nu}^{\prime}(q+),-B_{\nu}^{\prime}(q-)\right)\right)=0 .
$$

Proof. Due to Lemmas 4.2 and 4.6, $B_{\nu}(q)=\tau_{\mu}(q)$. Take $\alpha<-B_{\nu}^{\prime}(q+)$ and set

$$
E_{n}=\left\{x \in \operatorname{supp} \nu \mid \nu(B(x, r))>r^{\alpha} \text { for } r<1 / n\right\} .
$$

Choose $\varepsilon>0$ and $t>0$ such that $\tau_{\mu}(q)-\varepsilon-\alpha t>B_{\nu}(q+t)$. This can be done for positive, but arbitrarily small, $\varepsilon$. Once $\varepsilon$ is chosen, consider the following sets

$$
G_{m}=\left\{x \in \operatorname{supp} \nu \mid \nu_{q}(B(x, r)) \leq C \nu(B(x, r))^{q} r^{\tau_{\mu}(q)-\varepsilon} \text { for } r \leq 1 / m\right\},
$$

where $C$ is the constant in Lemma 4.6. Due to this lemma, $\bigcup_{m \geq 1} G_{m}$ has full $\nu_{q^{-}}$ measure.

Let $F$ be a subset of $E_{n}$. For any $\theta<\min (1 / n, 1 / m)$, consider a centered $\theta$-cover $\left\{B\left(x_{j}, r_{j}\right)\right\}$ of $F \cap G_{m}$ which splits in $\beta$ packings. 
We have, if $\nu_{q}^{*}$ stands for the outer measure associated with $\nu_{q}$,

$$
\begin{aligned}
\nu_{q}^{*}\left(F \cap G_{m}\right) & \leq \sum \nu_{q}\left(B\left(x_{j}, r_{j}\right)\right) \leq C \sum \nu\left(B\left(x_{j}, r_{j}\right)\right)^{q} r_{j}^{\tau_{\mu}(q)-\varepsilon} \\
& \leq C \sum \nu\left(B\left(x_{j}, r_{j}\right)\right)^{q+t} r_{j}^{\tau_{\mu}(q)-\varepsilon-\alpha t} \nu\left(B\left(x_{j}, r_{j}\right)\right)^{-t} r_{j}^{\alpha t} \\
& \leq C \sum \nu\left(B\left(x_{j}, r_{j}\right)\right)^{q+t} r_{j}^{\tau_{\mu}(q)-\varepsilon-\alpha t} \\
& \leq \beta C \overline{\mathcal{P}}_{\nu, \theta}^{q+t, \tau_{\mu}(q)-\varepsilon-\alpha t}(F) .
\end{aligned}
$$

This means that $\nu_{q}^{*}(F) \leq C \overline{\mathcal{P}}_{\nu}^{q+t, \tau_{\mu}(q)-\varepsilon-\alpha t}(F)$, and

$$
\nu_{q}\left(E_{n}\right) \leq \mathcal{P}_{\nu}^{q+t, \tau_{\mu}(q)-\varepsilon-\alpha t}\left(E_{n}\right) \leq \mathcal{P}_{\nu}^{q+t, \tau_{\mu}(q)-\varepsilon-\alpha t}(\operatorname{supp} \nu)
$$

But this last quantity equals 0 because $\tau_{\mu}(q)-\varepsilon-\alpha t \geq B_{\nu}(q+t)$. Therefore

$$
\nu_{q}\left(\left\{x \in \operatorname{supp} \nu \mid \limsup _{r \searrow 0} \frac{\log \nu(B(x, r))}{\log r}<\alpha\right\}\right)=\nu_{q}\left(\bigcup E_{n}\right)=0 .
$$

Thus we proved the equality

$$
\nu_{q}\left(\left\{x \in \operatorname{supp} \nu \mid \limsup _{r \searrow 0} \frac{\log \nu(B(x, r))}{\log r}<-B_{\nu}^{\prime}(q+)\right\}\right)=0 .
$$

The equality

$$
\nu_{q}\left(\left\{x \in \operatorname{supp} \nu \mid \limsup _{r \searrow 0} \frac{\log \nu(B(x, r))}{\log r}>-B_{\nu}^{\prime}(q-)\right\}\right)=0 .
$$

is proven in a similar way.

THEOREM 4.8. Under the same hypotheses as in Lemma 4.6, we have

$$
\operatorname{dim} X_{\nu}\left(-B_{\nu}^{\prime}(q+),-B_{\nu}^{\prime}(q-)\right) \geq \begin{cases}\tau_{\mu}^{*}\left(-B_{\nu}^{\prime}(q+)\right) & \text { if } q \geq 0 \\ \tau_{\mu}^{*}\left(-B_{\nu}^{\prime}(q-)\right) & \text { if } q \leq 0\end{cases}
$$

Proof. Due to Lemma 4.7, $\nu_{q}$ is carried by $X_{\nu}\left(-B_{\nu}^{\prime}(q+),-B_{\nu}^{\prime}(q-)\right)$. On the other hand, due to Lemma 4.6, for $\nu_{q}$-almost every $x$, for $\varepsilon>0$, and for $r$ small enough, one has

$$
\frac{\log \nu_{q}(B(x, r))}{\log r} \geq \frac{\log C}{\log r}+q \frac{\log \nu(B(x, r))}{\log r}+\tau_{\mu}(q)-\varepsilon .
$$

One concludes by using the Billingsley lemma [8].

Corollary 4.9. Let $q \in \mathbb{R}$. Assume there exists a Frostman measure $\mu_{q}$ for $\mu$ at $\left(q, \tau_{\mu}(q)\right)$. If $\tau^{\prime}(q)$ exists and if $\mu$ fulfills the $N B C$ for $q$, then both measures $\mu$ and $\nu=\gamma_{*}(\mu)$ satisfy the multifractal formalism at $q$ and one has $b_{\nu}(q)=B_{\nu}(q)=$ $\Lambda_{\nu}(q)=b_{\mu}(q)=B_{\mu}(q)=\Lambda_{\mu}(q)=\tau_{\mu}(q)$.

By careful analysis of the steps which lead to the previous theorem and its corollary, and a few modification, one can prove the following result. 
Theorem 4.10. Let $q \in \mathbb{R}$. Assume there exists a non-zero measure $\mu_{q}$ on $\partial \mathcal{A}^{*}$ and a function $C_{q}$ on $\mathcal{A}^{*}$ such that $\mu_{q}(w) \leq C_{q}(w) \mu(w)^{q} c^{-|w| \tau_{\mu}(q)}$ for all $w \in \mathcal{A}^{*}$ such that $\mu(w)>0$. If $\tau^{\prime}(q)$ exists and if, for all $\varepsilon>0$, there exists $\eta>0$ such that, we have

$$
\sum_{n>0} c^{-n\left(\tau_{\mu}(q)+\eta \varepsilon\right)} \sum_{\substack{v, w \in \mathcal{A}^{n} \\ \delta(v, w) \leq c^{\prime}}} \mu(v)^{-\eta q} C_{q}(w)^{1+\eta} \mu(w)^{(1+\eta) q}<+\infty
$$

(where $c^{\prime}=3$ if $q<0, c^{\prime}=4 c+2$ otherwise), then both measures $\mu$ and $\nu=\gamma_{*}(\mu)$ obey the multifractal formalism at $q$, and $b_{\nu}(q)=B_{\nu}(q)=\Lambda_{\nu}(q)=b_{\mu}(q)=B_{\mu}(q)=$ $\Lambda_{\mu}(q)=\tau_{\mu}(q)$.

One can remark that the conjunction of the existence of a Gibbs measure and of the NBC is stronger than the hypothesis of this last theorem, which therefore can be called weak NBC.

REMARK. [The case of higher dimension] We stated and proved these comparison theorems in a one dimensional setting in order not to deal with too complicated notations. But these results hold for measures on $\mathbb{R}^{d}$. Here are the few modifications to be made to accomodate this case.

This time, the alphabet is of the form $\mathcal{A}^{d}$. So each node $w$ of the tree $\left(\mathcal{A}^{d}\right)^{*}$ can be viewed as a collection $\left(w_{1}, w_{2}, \ldots, w_{d}\right)$ of words on $\mathcal{A}$. An element $x$ of $\partial\left(\mathcal{A}^{d}\right)^{*}$ can be identified to $\left(x_{1}, x_{2}, \ldots, x_{d}\right) \in\left(\partial \mathcal{A}^{*}\right)^{d}$ and a mapping, which we again call $\gamma$, from $\partial\left(\mathcal{A}^{d}\right)^{*}$ to $\mathbb{R}^{d}$ is defined by $\gamma(x)=\left(\gamma\left(x_{1}\right), \gamma\left(x_{2}\right), \ldots, \gamma\left(x_{d}\right)\right)$. Similarly, the distance of two nodes $v=\left(v_{1}, v_{2}, \ldots, v_{d}\right)$ and $w=\left(w_{1}, w_{2}, \ldots, w_{d}\right)$ of the same generation, again denoted by $\delta$, is $\delta(v, w)=\max \left\{\delta\left(v_{1}, w_{1}\right), \delta\left(v_{2}, w_{2}\right), \ldots, \delta\left(v_{d}, w_{d}\right)\right\}$. At last, the value of $c^{\prime}$ is unchanged. Then, as already said, the previous results hold, with the same proofs, if $\mu$ is a measure on $\partial\left(\mathcal{A}^{d}\right)^{*}$ and $\nu=\gamma_{*}(\mu)$.

5. Quasi-Bernoulli measures. A probability measure on $\partial \mathcal{A}^{*}$ is said to be quasi-Bernoulli if there exists $C>0$ such that, for any $v$ and $w$ in $\mathcal{A}^{*}$, one has

$$
\frac{1}{C} \mu(v) \mu(w) \leq \mu(v w) \leq C \mu(v) \mu(w) .
$$

The multinomial measures constitute a paradigm of such measures. They are very special elements of a larger family. Indeed, denote by $S$ the shift operation on $\partial \mathcal{A}^{*}$. Due to its construction [10], any Gibbs measure with Hölder potential on the dynamical system $\left(\partial \mathcal{A}^{*}, S\right)$ is quasi-Bernoulli. Moreover, any quasi-Bernoulli measure is equivalent to an ergodic quasi-Bernoulli measure (see [20]).

It turns out from $[28,29,11]$ that for every $q \in \mathbb{R}$ there exists a probability measure $\mu_{q}$ and a constant $C_{q}>0$, such that, for every $v \in \mathcal{A}^{*}$ such that $\mu(v) \neq 0$, one has

$$
\frac{1}{C_{q}} \mu(v)^{q} c^{-\tau_{\mu}(q)|v|} \leq \mu_{q}(v) \leq C_{q} \mu(v)^{q} c^{-\tau_{\mu}(q)|v|} .
$$

Moreover, the function $\tau_{\mu}$ is differentiable [20]. As a consequence, the first two assumptions in Corollary 4.9 are fulfilled for every $q \in \mathbb{R}$.

Clearly, a quasi-Bernoulli probability measure $\mu$ on $\partial \mathcal{A}^{*}$ has an atom if and only if $\mu(j)=1$ for some $j \in \mathcal{A}^{1}$, in which case $\mu=\delta_{j j \ldots j \ldots}$. We discard this case and prove the following result. 
THEOREM 5.1. Let $\mu$ be a continuous quasi-Bernoulli measure on $\partial \mathcal{A}^{*}$. Then both measures $\mu$ and $\nu=\gamma_{*}(\mu)$ obey the multifractal formalism everywhere and one has $b_{\nu}=B_{\nu}=\Lambda_{\nu}=b_{\mu}=B_{\mu}=\Lambda_{\mu}=\tau_{\mu}$.

This result is established without using the NBC in [7] under the strong hypothesis: for all $n \geq 1$, if $a, b \in \mathcal{A}^{n}$ and $\delta(a, b)=1$ then $\mu(a) \mu(b)=0$. In particular, the case $c=2$ is excluded and in the case $c=3 \mu$ is a Dirac mass.

Proof. We begin by a preliminary remark: if $v$ and $w$ are words of length $n$, and if $\dot{v}$ and $\dot{w}$ stand for their prefixes of length $n-1$, then $\delta(\dot{v}, \dot{w})>k$ implies $\delta(v, w)>c k$. It results that, given two integers $n \geq m>0$ and two words $v$ and $w$ in $\mathcal{A}^{n}$ such that $c^{m-1}<\delta(v, w) \leq c^{m}$, there exists two prefixes $\bar{v}$ and $\bar{w}$ of $v$ and $w$ respectively of common length $n-m$ such that $\delta(\bar{v}, \bar{w}) \leq 1$.

It results from (5.1) and the above remark that, for a quasi-Bernoulli measure to fulfill the NBC it is enough that for all $\varepsilon>0$, there exists $\eta>0$ such that

$$
\sum_{n>0} c^{-n\left(\tau_{\mu}(q)+\eta \varepsilon\right)} \sum_{\substack{v, w \in \mathcal{A}^{n} \\ \delta(v, w) \leq 1}} \mu(v)^{-\eta q} \mu(w)^{(1+\eta) q}<+\infty .
$$

Due to the existence of the Gibbs measure $\mu_{q}$ this reduces to

$$
\sum_{n>0} c^{-n\left(\tau_{\mu}(q)+\eta \varepsilon\right)} \sum_{\substack{v, w \in \mathcal{A}^{n} \\ \delta(v, w)=1}} \mu(v)^{-\eta q} \mu(w)^{(1+\eta) q}<+\infty .
$$

Define $\tilde{\mathcal{A}}=\{0 \leq j \leq c-2 \mid \mu(j) \mu(j+1) \neq 0\}$.

Define $\rho_{k}$ to be the word consisting of $k$ consecutive zeros and $\lambda_{k}$ to be the word consisting of $k$ consecutive $c-1$ (considered as a letter from the alphabet $\{0,1,2, \ldots, c-1\})$.

By (5.1), for $n \geq 1$, a representation of the set of pairs $(v, w)$ in $\mathcal{A}^{n}$ such that $\mu(v) \mu(w) \neq 0$ and $\imath(w)=\imath(v)+1$ is as follows:

$$
\bigcup_{k=0}^{n-1} \bigcup_{\substack{u \in \mathcal{A}^{n-1-k} \\ \mu(u) \neq 0}}\left\{\left(u \cdot j \cdot \lambda_{k}, u \cdot(j+1) \cdot \rho_{k}\right) \mid j \in \tilde{\mathcal{A}}\right\}
$$

if $\mu(0) \mu(c-1) \neq 0$ and

$$
\bigcup_{\substack{u \in \mathcal{A}^{n-1} \\ \mu(u) \neq 0}}\{(u \cdot j, u \cdot(j+1)) \mid j \in \tilde{\mathcal{A}}\}
$$

otherwise.

We end the proof when $\mu(0) \mu(c-1) \neq 0$. The other case is simpler. We have to prove that for every $\varepsilon>0$, there exists $\eta>0$ such that

$$
\begin{aligned}
S_{q, \varepsilon}(\eta)= & \sum_{n \geq 1} c^{-n\left(\tau_{\mu}(q)+\eta \varepsilon\right)} \sum_{\substack { k=0 \\
\begin{subarray}{c}{u \in \mathcal{A}^{n-1-k} \\
\mu(u) \neq 0{ k = 0 \\
\begin{subarray} { c } { u \in \mathcal { A } ^ { n - 1 - k } \\
\mu ( u ) \neq 0 } }\end{subarray}}^{n-1} \sum \\
& \sum_{j \in \tilde{\mathcal{A}}} \mu\left(u \cdot j \cdot \lambda_{k}\right)^{q} \frac{\mu\left(u \cdot j \cdot \lambda_{k}\right)^{q \eta}}{\mu\left(u \cdot(j+1) \cdot \rho_{k}\right)^{q \eta}}+\mu\left(u \cdot(j+1) \cdot \rho_{k}\right)^{q} \frac{\mu\left(u \cdot(j+1) \cdot \rho_{k}\right)^{q \eta}}{\mu\left(u \cdot j \cdot \lambda_{k}\right)^{q \eta}}
\end{aligned}
$$


is finite.

Fix $\varepsilon>0$. Let $\eta$ be a positive number to be chosen later on. By (5.1),

$$
\begin{gathered}
\mu\left(u \cdot j \cdot \lambda_{k}\right)^{q} \frac{\mu\left(u \cdot j \cdot \lambda_{k}\right)^{q \eta}}{\mu\left(u \cdot(j+1) \cdot \rho_{k}\right)^{q \eta}}+\mu\left(u \cdot(j+1) \cdot \rho_{k}\right)^{q} \frac{\mu\left(u \cdot(j+1) \cdot \rho_{k}\right)^{q \eta}}{\mu\left(u \cdot j \cdot \lambda_{k}\right)^{q \eta}} \leq \\
C(q, \eta) \mu(u)^{q}\left[\mu\left(\lambda_{k}\right)^{q} \frac{\mu\left(\lambda_{k}\right)^{q \eta}}{\mu\left(\rho_{k}\right)^{q \eta}}+\mu\left(\rho_{k}\right)^{q} \frac{\mu\left(\rho_{k}\right)^{q \eta}}{\mu\left(\lambda_{k}\right)^{q \eta}}\right]
\end{gathered}
$$

for some constant $C(q, \eta)$. Therefore

$$
S_{q, \varepsilon}(\eta) \leq(c-1) C(q, \eta) \sum_{n \geq 1} c^{-n\left(\tau_{\mu}(q)+\eta \varepsilon\right)} f_{n}(q, \eta)
$$

with

$$
f_{n}(q, \eta)=\sum_{k=0}^{n-1} c^{(n-1-k) \tau_{\mu, n-1-k}(q)}\left[\mu\left(\lambda_{k}\right)^{q} \frac{\mu\left(\lambda_{k}\right)^{q \eta}}{\mu\left(\rho_{k}\right)^{q \eta}}+\mu\left(\rho_{k}\right)^{q} \frac{\mu\left(\rho_{k}\right)^{q \eta}}{\mu\left(\lambda_{k}\right)^{q \eta}}\right]
$$

where

$$
\tau_{\mu, k}(q)=\frac{1}{k} \log _{c} \sum_{u \in \mathcal{A}^{k}}^{*} \mu(u)^{q}
$$

By using (5.2) we get

$$
\frac{1}{C_{q}} c^{k \tau_{\mu}(q)} \leq c^{k \tau_{\mu, k}(q)} \leq C_{q} c^{k \tau_{\mu}(q)}
$$

Then (5.2) and (5.7) yield

$$
f_{n}(q, \eta) \leq C_{q}^{2+2 \eta} c^{(n-1) \tau_{\mu}(q)} \sum_{k=0}^{n-1}\left[\mu_{q}\left(\lambda_{k}\right) \frac{\mu_{q}\left(\lambda_{k}\right)^{\eta}}{\mu_{q}\left(\rho_{k}\right)^{\eta}}+\mu_{q}\left(\rho_{k}\right) \frac{\mu_{q}\left(\rho_{k}\right)^{\eta}}{\mu_{q}\left(\lambda_{k}\right)^{\eta}}\right] .
$$

By construction $\mu_{q}$ has no atoms and is quasi-Bernoulli. Consequently, by the sub-multiplicativity property (up to a multiplicative constant) of $\mu_{q}$, both $\lim _{n \rightarrow \infty} \frac{1}{n} \log \mu_{q}\left(\rho_{n}\right)$ and $\lim _{n \rightarrow \infty} \frac{1}{n} \log \mu_{q}\left(\lambda_{n}\right)$ exist and are negative. Therefore if $\eta$ is small enough (notice that $\eta$ does not depend on $\varepsilon$ ), one has

$$
M_{q}(\eta)=\sup _{k \in \mathbb{N}}\left[\mu_{q}\left(\rho_{k}\right) \frac{\mu_{q}\left(\rho_{k}\right)^{\eta}}{\mu_{q}\left(\lambda_{k}\right)^{\eta}}+\mu_{q}\left(\lambda_{k}\right) \frac{\mu_{q}\left(\lambda_{k}\right)^{\eta}}{\mu_{q}\left(\rho_{k}\right)^{\eta}}\right]<\infty
$$

and, by (5.8),

$$
f_{n}(q, \eta) \leq C_{q}^{2+2 \eta} M_{q}(\eta) n c^{(n-1) \tau_{\mu}(q)}
$$

Finally by (5.6)

$$
S_{q, \varepsilon}(\eta) \leq(c-1) C(q, \eta) C_{q}^{2+2 \eta} M_{q}(\eta) c^{-\tau_{\mu}(q)} \sum_{n \geq 1} n c^{-n \eta \varepsilon}<\infty .
$$


6. Statistically self-similar measures. In this section, we consider the random measures introduced by B. Mandelbrot in [24]. Up to now, their multifractal analysis has been mostly performed in the setting of the tree of $c$-adic intervals (see [22, 21, 14, 30, 2, 3]). Nevertheless, Arbeiter and Patzschke [1] obtained a result in the same spirit as ours under strong assumptions without setting a general frame. To be more specific, they compute the Hausdorff dimension of the level sets of the local centered Hölder exponent, for each $\alpha$ with probability one, for a Mandelbrot cascade on the attractor of an IFS satisfying the OSC.

Mandelbrot measures do not satisfy the NBC. This motivated the consideration of the weak NBC (Theorem 4.10).

For the sake of simplicity, we only deal with the so-called canonical multiplicative cascades. Let us recall a construction of these measures.

Fix $W$ a non-negative random variable. Assume that $W$ is not almost surely constant and that $\mathbb{E}(W)=1 / c$.

Define the function $\tilde{\tau}(q)=1+\log _{c} \mathbb{E}\left(1_{\{W>0\}} W^{q}\right)$ for $q \in \mathbb{R}$.

In order to avoid technicalities, unessential to our purpose, we assume that $W$ is positive and that $\widetilde{\tau}(q)$ is finite for any $q \in \mathbb{R}$.

Let $\left(W_{w}\right)_{w \in \mathcal{A}^{*}}$ be a sequence of independent copies of $W$. For every $n \geq 1$, consider the random measure $\mu_{n}$ whose density with respect to the uniform probability measure on $\partial \mathcal{A}^{*}$ is locally constant and equals

$$
c^{n} W_{w_{1}} W_{w_{1} w_{2}} \ldots W_{w_{1} w_{2} \ldots w_{n}}
$$

on the cylinder $w=w_{1} w_{2} \ldots w_{n}$. With probability one, the sequence $\mu_{n}$ converges weakly to a measure $\mu$ as $n$ goes to infinity. Moreover, if $\widetilde{\tau}^{\prime}(1)<0$, one has $\mu \neq 0$ (see [23]).

Then, define $J=\left\{q \in \mathbb{R} ; \tilde{\tau}^{*}\left(-\tilde{\tau}^{\prime}(q)\right)>0\right\}$. It follows from Theorem $8(i v)$ in [3] that $\tau_{\mu}=\tilde{\tau}$ on $J$.

THEOREM 6.1. With probability one, both measures $\mu$ and $\nu=\gamma_{*}(\mu)$ satisfy the multifractal formalism on $J$ and $b_{\nu}=B_{\nu}=\Lambda_{\nu}=b_{\mu}=B_{\mu}=\Lambda_{\mu}=\tau_{\mu}=\widetilde{\tau}$ on $J$.

Proof. For every $q \in J, v \in \mathcal{A}^{*}$ and $n \geq 1$, define

$$
Y_{q, n}(v)=c^{-n \tilde{\tau}(q)} \sum_{w_{1} \ldots w_{n} \in \mathcal{A}^{n}} W_{v w_{1}}^{q} W_{v w_{1} w_{2}}^{q} \ldots W_{v w_{1} w_{2} \ldots w_{n}}^{q} .
$$

It follows from Corollary 5 in [3] that, with probability one, for all $v \in \mathcal{A}^{*}$ and all $q \in J$, the limit $Y_{q}(v)=\lim _{n \rightarrow \infty} Y_{q, n}(v)$ exists. Moreover, with probability one, for all $q \in J$, the mapping $\mu_{q}$ defined on $\partial \mathcal{A}^{*}$ by

$$
\mu_{q}(v)=c^{-|v| \widetilde{\tau}(q)} Y_{q}(v) \prod_{j=1}^{|v|} W_{v_{1} \cdots v_{j}}^{q}
$$

defines a measure (notice that $\mu_{1}=\mu$ ); all the measures $\mu_{q}$ have $\partial \mathcal{A}^{*}$ as support and for all $v \in \mathcal{A}^{*}$ and $q \in J$,

$$
\mu_{q}(v)=C_{q}(v) \mu(v)^{q} c^{-|v| \tilde{\tau}(q)}
$$


with

$$
C_{q}(v)=\frac{Y_{q}(v)}{Y_{1}^{q}(v)}
$$

Since $\tau_{\mu}=\widetilde{\tau}$ on $J$, the result will be a consequence of Theorem 4.10 if we show that for every non trivial compact subinterval $K$ of $J$, with probability one, for all $q \in K$, for all $\varepsilon>0$, there exists $\eta>0$ such that

$$
\sum_{n \geq 1} c^{-n(\widetilde{\tau}(q)+\eta \varepsilon)} \sum_{\substack{v, w \in \mathcal{A}^{n} \\ \delta(v, w) \leq c^{\prime}}} \mu(v)^{-\eta q} C_{q}(w)^{1+\eta} \mu(w)^{(1+\eta) q}<+\infty
$$

i.e.

$$
\sum_{n \geq 1} c^{-n(\widetilde{\tau}(q)+\eta \varepsilon)} f_{n, \varepsilon, \eta}(q)<\infty
$$

where

$$
f_{n, \varepsilon, \eta}(q)=\sum_{\substack{v, w \in \mathcal{A}^{n} \\ \delta(v, w) \leq c^{\prime}}} Y_{1}(v)^{-\eta q} Y_{q}(w)^{1+\eta} \prod_{k=1}^{n} W_{v_{1} \ldots v_{k}}^{-\eta q} W_{w_{1} \ldots w_{k}}^{(1+\eta) q}
$$

Fix such a compact $K$. It turns out that it suffices to show that for every $\varepsilon>0$, if $\eta>0$ is small enough, then

$$
\left\{\begin{array}{l}
\sum_{n \geq 1} \sup _{q \in K} n c^{-n(\widetilde{\tau}(q)+\eta \varepsilon)} \mathbb{E}\left(f_{n, \varepsilon, \eta}(q)\right)<\infty \\
\sum_{n \geq 1} \sup _{q \in K} c^{-n(\tilde{\tau}(q)+\eta \varepsilon)} \mathbb{E}\left(\left|f_{n, \varepsilon, \eta}^{\prime}(q)\right|\right)<\infty
\end{array}\right.
$$

(see the proof of Corollary 1 in [3] for a more detailed similar argument).

It follows from Lemma 6 in [3] that for $\eta$ small enough

$$
C_{K}(\eta)=\sup _{\substack{q \in K, n \geq 1, v, w \in \mathcal{A}^{n}}} \mathbb{E}\left(\left|\frac{d}{d q}\left(Y_{1}(v)^{-\eta q} Y_{q}(w)^{1+\eta}\right)\right|\right)+\mathbb{E}\left(Y_{1}(v)^{-\eta q} Y_{q}(w)^{1+\eta}\right)<\infty
$$

and

$$
C_{K}^{\prime}(\eta)=\sup _{\substack{q \in K, n \geq 1, v, w \in \mathcal{A}^{n}}} \frac{\mathbb{E}\left(\left|\frac{d}{d q} W_{v}^{-\eta q} W_{w}^{(1+\eta) q}\right|\right)}{\mathbb{E}\left(W_{v}^{-\eta q} W_{w}^{(1+\eta) q}\right)}<\infty .
$$

By taking into account the fact that the $W$ s are mutually independent, we get

$$
\mathbb{E}\left(\left|f_{n, \varepsilon, \eta}^{\prime}(q)\right|\right) \leq C_{K}(\eta)\left(1+n C_{K}^{\prime}(\eta)\right) g_{n, \varepsilon, \eta}(q)
$$

where

$$
g_{n, \varepsilon, \eta}(q)=\sum_{\substack{v, w \in \mathcal{A}^{n} \\ \delta(v, w) \leq c^{\prime}}} \prod_{k=1}^{n} \mathbb{E}\left(W_{v_{1} \cdots v_{k}}^{-\eta q} W_{w_{1} \cdots w_{k}}^{(1+\eta) q}\right)
$$


Also, we have

$$
\mathbb{E}\left(f_{n, \varepsilon, \eta}(q)\right) \leq C_{K}(\eta) g_{n, \varepsilon, \eta}(q) .
$$

By using a reduction similar to the one made in the beginning of Section 5 , we can assume without loss of generality that in the sums over $\left\{v, w \in \mathcal{A}^{n} ; \delta(v, w) \leq c^{\prime}\right\}$, there are only pairs $(v, w)$ for which $\delta(v, w) \leq 1$. Then, by using (5.4), we get

$$
g_{n, \varepsilon, \eta}(q)=c^{n \tilde{\tau}(q)}+h_{n, \varepsilon, \eta}(q)
$$

where

$$
\begin{aligned}
& h_{n, \varepsilon, \eta}(q)=(c-1) \sum_{k=0}^{n-1} c^{k}\left(\mathbb{E}\left(W^{q}\right)\right)^{k}\left(\mathbb{E}\left(W^{-\eta q}\right) \mathbb{E}\left(W^{(1+\eta) q}\right)\right)^{n-k} \\
= & (c-1)\left(\mathbb{E}\left(W^{-\eta q}\right) \mathbb{E}\left(W^{(1+\eta) q}\right)\right)^{n} \sum_{k=0}^{n-1}\left[\frac{c \mathbb{E}\left(W^{q}\right)}{\mathbb{E}\left(W^{-\eta q}\right) \mathbb{E}\left(W^{(1+\eta) q}\right)}\right]^{k} \\
\leq & c^{1+n\left(-2+\tilde{\tau}(q)+\widetilde{\tau}(0)+\eta q\left(\tilde{\tau}^{\prime}(q)-\tilde{\tau}^{\prime}(0)\right)+\eta \varepsilon_{q}(\eta)\right)} \\
& \times \sum_{k=0}^{n-1} c^{k\left(2-\tilde{\tau}(0)-\eta q\left(\tilde{\tau}^{\prime}(q)-\tilde{\tau}^{\prime}(0)\right)+\eta \varepsilon_{q}(\eta)\right)} \\
\leq & \frac{c^{1+n\left(\tilde{\tau}(q)+\eta \varepsilon_{q}(\eta)\right)}}{c^{2-\tilde{\tau}(0)-\eta q\left(\tilde{\tau}^{\prime}(q)-\tilde{\tau}^{\prime}(0)\right)+\eta \varepsilon_{q}(\eta)}-1},
\end{aligned}
$$

with $\varepsilon_{q}(\eta) \rightarrow 0$ uniformly on $K$ when $\eta \rightarrow 0$. Then, it is easily seen that (6.4) holds if $\eta$ is small enough.

\section{REFERENCES}

[1] M. Arbeiter ANd N. PATzSChKe, Random self-similar multifractals, Math. Nachr., 181 (1996), pp. 5-42.

[2] J. BARRAL, Moments, continuité et analyse multifractale des martingales de Mandelbrot, Probab. Theory Relat. Fields, 113 (1999), pp. 535-569.

[3] J. BARral, Continuity of the multifractal spectrum of a random statistically self-similar measures, J. Theor. Probab., 13 (2000), pp. 1027-1060.

[4] T. BEDFORD, Applications of dynamical systems theory to fractals: a study of cookie-cutter Cantor sets, In Fractal Geometry and Analysis. Eds. Belair J. and Dubuc S., Kluwer Academic Publishers, 1991.

[5] F. Ben NAsR, Analyse multifractale de mesures, C. R. Acad. Sci. Paris, 319, Série I (1994), pp. 807-810.

[6] F. Ben NASR, I. Bhouri, AND Y. Heurteaux, The validity of the multifractal formalism: results and examples, Adv. in Math, 165 (2002), pp. 264-284.

[7] I. BHOURI, Une condition de validité du formalisme multifractal pour les mesures, Thèse de Doctorat. Tunis, 1999.

[8] P. Billingsley, Ergodic Theory and Information, J. Wiley, New York, 1965.

[9] T. BohR AND D. RAND, The entropy function for characteristic exponents, Physica, 25D (1987), pp. 387-398.

[10] R. BOWEN, Equilibrium states and the ergodic theory of Anosov Diffeomorphisms, Lectures Notes in Mathematics, 470, Dold A. and Eckmann B. Eds., Springer.

[11] G. Brown, G. Michon, AND J. PEYrière, On the multifractal analysis of measures, J. Stat. Phys., 66 (1992), No. 3/4, pp. 775-790.

[12] H. CHERnOFF, A measure of asymptotic efficiency for tests of a hypothesis based on the sum of observations, Ann. Math. Statist, 23 (1952), pp. 493-507.

[13] P. Collet, J.L. Lebowitz, AND A. Porzio, The dimension spectrum of some dynamical systems, J. Stat. Phys, 47 (1987), pp. 609-644. 
[14] P. Collet, F. Koukiou, Large deviations for multiplicative chaos, Commun. Math. Phys., 147 (1992), pp. 329-342.

[15] G.A. Edgar AND R.D. MAUldin, Multifractal decomposition of digraph recursive fractals, Proc. London Math. Soc., 65 (1992), pp. 604-628.

[16] K.J. FALCONER, The multifractal spectrum of statistically self-similar measures, J. Theor. Prob., 7 (1994), pp. 681-702.

[17] U. FRISCH AND G. PARISI, Fully developped turbulence and intermittency in turbulence, and predictability in geophysical fluid dynamics and climate dymnamics, International school of physics "Enrico Fermi", course 88, edited by M. Ghil, North Holland (1985), p. 84.

[18] T.C. Halsey, M.H. Jensen, L.P. Kadanoff, I. Procaccia, and B.I. Shraiman, Fractal measures and their singularities: the characterisation of strange sets, Phys. Rev. A, 33 (1986), p. 1141.

[19] H.G.E. HentSChel AND I. Procaccia, The infinite number of generalized dimensions of fractals and strange attractors, Physica, 8D (1983), p. 435.

[20] Y. Heurteaux, Estimations de la dimension inférieure et de la dimension supérieure des mesures, Ann. Inst. Henri Poincaré, 34 (1998), pp. 309-338.

[21] R. Holley AND E.C. WAymire, Multifractal dimensions and scaling exponents for strongly bounded random cascades, Ann. Appl. Probab., 2 (1992), pp. 819-845.

[22] J.-P. Kahane, Produits de poids aléatoires et indépendants et applications, in Fractal Geometry and Analysis, J. Bélair and S. Dubuc (eds.), 1991, pp. 277-324.

[23] J.-P. KAhane et J. Peyrière, Sur certaines martingales de Benô̂t Mandelbrot. Adv. in Math., 22 (1976), pp. 131-145.

[24] B.B. MANDELBROT, Intermittent turbulence in self-similar cascades: divergence of high moments and dimension of the carrier, J. Fluid Mech., 62 (1974), pp. 331-358.

[25] B.B. MANDELBRot, Multifractal measures, especially for the geophysicist, Annual reviews of materials sciences, 19 (1989), pp. 514-516.

[26] B.B. MANDELBRot, A class of multifractal measures with negative (latent) value for the dimension $f(\alpha)$, Fractals: physical origin and properties (Erice, 1988). Edited by Luciano Pietronero, New York: Plenum, 1989.

[27] B.B. MANDELBROT, Two meanings of multifractality, and the notion of negative fractal dimension, Soviet-American chaos meeting (Woods Hole, 1989). Edited by Kenneth Ford and David Campbell, American Institute of Physics, 1990.

[28] G. Michon, Une construction des mesures de Gibbs sur certains ensembles de Cantor, C. R. Acad. Sc. Paris, 308 (1989), pp. 315-318.

[29] G. Michon, Mesures de Gibbs sur les Cantor Réguliers, Ann. Inst. Henri Poincaré, Phys. Théor., 58:3 (1993), pp. 267-285.

[30] G.M. MolChAN, Scaling exponents and multifractal dimensions for independent random cascades, Commun. Math. Phys., 179 (1996), pp. 681-702.

[31] L. OLSEN, Random geometrically graph directed self-similar multifractals, Pitman Res. Notes Math. Ser., 307 (1994).

[32] L. OlsEN, A multifractal formalism. Adv. Math., 116 (1995), pp. 92-195.

[33] J. PeYrière, Multifractal measures, In Probabilistic and Stochastic Methods in Analysis (Proceedings of the NATO ASI, Il Ciocco 1991). Ed. J. Byrnes, Kluwer Academic Publishers, 1992.

[34] D.A. RAND, The singularity spectrum $f(\alpha)$ for cookie-cutters, Ergod. Th. \& Dynam. Sys., 9 (1989), pp. 527-541.

[35] X. SAint RAYmond and C. TRicot, Packing regularity of sets in $n$-space, Math. Proc. Cambridge Philos. Soc, 288 (1985), pp. 679-699.

[36] C. TRICOT, Two definitions of fractional dimension, Math. Proc. Cambridge Philos. Soc., 91 (1982), pp. 57-74. 
